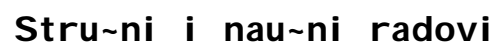

Zna-aj probl ema karci noma pl u\}a

u`enskoj popul aci ji Rasi nskog okr uga

V. Krst i $\},{ }^{1}$ Q. Crn-evi \}-Radovi $\}^{2}$

\title{
Importance of the Problem of Lung Cancer in the Woman's Population of the Rasinian Region in Serbia
}

Krstic V., M. D., D. P. H., Lj. Crncevic-Radovic, M. D., D. P. H.

Sa' et ak. Ci q ovog i st ra` i vawa je ut vr|i vawe u-est al ost i obol evawa i umi rawa od karci noma pl u\}a u 'enskoj popul aci ji Rasi nskog okr uga u peri odu od 1995. do 2000. godi ne.

U ovom i st ra` i vawu pri mewena je deskri pt i vna epi demi ol o\{ ka st udi ja karci noma pl u\}a u 'enskoj popul aci ji Rasi nskog okr uga $u$ dat om peri odu.

Model i I i near ni h t rendova za 'ene Rasi nskog okr uga za si rovu i svet sku st opu i nci dence $i$ mort al i t et a od kar ci noma pl u\}a ukazuju na post ojawe $t$ endenci je st al nog porast a vrednost i ovi h st opa $u$ pomenut oj popul aci ji u posmat ranomperi odu.

Si rova st opa i nci dence karci noma pl u\}a u 'enskoj popul aci ji Rasi nskog okruga pokazuje st at i st i - ki zna ajan t rend porast a ( $p$ je mawe od 0,01$)$, kao i svet ska st opa ( $p$ je mawe od 0,05 ).

Si rova st opa mort al i t et a od karci noma pl u\}a u ovoj popul aci ji pokazuje st at i st $\mathrm{i}-\mathrm{ki}$ zna-ajan $\mathrm{t}$ rend porast a ( $p$ je mawe od 0,05 ), kao i svet ska st opa ( $p$ je mawe od $0,01)$.

Kqune re-i: Karci nom pl u\}a, ‘enska popul aci ja.
Summary. The goal of this research is the identification of the frequency of incidence and mortality of the lung cancer in the vvoman's population of the Rasinian region in the period 1995 to 2000.

Descriptive epidemiology study of the lung's cancer in the womans population of the Rasinian region in this period is applied in this research.

Models of the linear trends for the women of the Rasinian region for the crudes and world's incidence and mortality rate of the lung cancer shows existence of the constant, increase tendency the values rates in observe population in this period.

Crude's rate of incidence of the lung cancer in the woman's population of the Rasinian region shows statisticly significant increase trend $(p$ is less than 0,001) just as vvorld's rate ( $p$ is less than 0,05).

Crude's rate of mortality of the lung cancer in this ${ }_{p}$ opulat,on shows statisticly significant increase trend ( $p$ is less than 0,05) just as world's rate ( $p$ is less than 0,01)..

Key words: Liung cancer, woman's population.

\footnotetext{
${ }^{1}$ Dr Vesna Krsti \}, speci jal i sta soci jal ne medi ci ne, Dom zdravqa „Dr Sava Stanojevi \}“, Trsteni k.

${ }^{2}$ Dr Q i qana Crn-evi \}-Radovi \}, speci jal i sta soci jal ne medi ci ne, I nsti tut za javno zdravqe Srbi je „Dr Mi I an J ovanovi \}-Batut", Beograd.
} 


\section{Uvod}

U Sr bi ji, danas. kar ci nomi su razl og smrti 22.000 qudi godi $\{$ we, $\{$ to predstavq a drugo mesto po I okal i zaci ji naj-e\{ \}i h uzroka smrti, odmah i za kar di ovaskul ar ni h bol esti . Novi ja stati st i ka ukazuje na -i weni cu da oko 35 odsto gral ana Srbi je umi re od mal i gni h bol est i , i I i wi hovi h posl edi ca. Vode\}e I okal i zaci je karci noma jesu: pl u\}a kod mu\{karaca, dojka i pl u\}a kod 'ena i kol or ektum kod oba pol a. ${ }^{1}$

Karci nom pl u\}a se odl i kuje vi sokom f rekvenci jom pojavq i vawa i i zra` enom di nami kom rasta i razvoja, kao i ni ski mstopama pet ogodi \{ weg pre' i vaq avawa u odnosu na dr uge mal i gni tete - u SA D-u je 13 odsto, a kod nas i spod sedam odsto. P rose no pre' i vq avawe u zavi snost i od hi stopatol o\{ kog ti pa jeste od \{ est meseci do dve godi ne od postavq awa di jagnoze.

Karci nom pl u\}a i ma strahovi t uti caj na mortal itet u SAD-u, sa regi strovani $h$ 156.900 sl u ajeva u 2000. godi ni, me| u mr \{ kar ci ma i 'enama kombi novano. Rak pl u\}a sada u-est vuje sa 14 odsto me| u novi msl u-ajevi ma obol evawa od karci noma i sa 28 odsto mel u svi msl u-ajevi ma smrti od karci noma godi $\{$ we u SAD-u. Stopa mortal i teta od raka pl u\}a nadma\{ i I a je onu i z 1987. godi ne od raka dojke, zbog -ega je kar ci nom pl u\}a danas vode\}a I okal i zaci ja me| u uzr oci ma smr ti od karci noma kod oba pol $a^{2}$.

Broj regi strovani h novoobol el i h od karci noma pl u\}a u Srbi ji 1995. godi ne i znosi o je bl i zu 4.000 hi qade, a broj umrl i h 3.682. U 2000. godi ni se o-ekuje da u Srbi ji umre preko 4200 osoba od ovog kar ci noma. Najve\}i porast smrtnosti od raka pl u\}a je na podru-ju Central ne Srbi je i Kosova i Metohi je, a najmawi na podr u-ju Vojvodi ne. Najve\}e standar di zovane stope mortal i teta su na podru-ju Vojvodi ne i u i ndustri jski m gradovi ma Central ne Srbi je (Beograd, [ abac, Loznica, Bor), a Trep-a na Kosovu i Metohi ji .

P ri kazani epi demi ol o\{ ki podaci ukazuju na to da postoji skri vena epi demi ja raka pl u\}a, \{ to zaht eva or gani zovanu prevenci ju u bor bi proti v ovog oboq ewa.

\section{Met od rada}

U ovom i stra' i vawu pri mewena je deskri pti vna epi demi ol o\{ ka st udi ja kar ci noma pl u\}a u 'enskoj popul aci ji Rasi nskog okruga u peri odu od 1995. do 2000. godi ne.

Osnovni predusl ov za kor ekt no i zvedeno epi demi ol o\{ ko-deskri pt i vno i stra' i vawe bol est i jeste poznavawe wene u-estal ost i i raspr ostrawenost i u odr el enoj, def i ni sanoj popul aci ji . To se posti e procenomparametara f rekvenci je i di stri buci je u-estal ost $i$ bol esti , u konkr etnom sl u aju stopa i nci dence karci noma pl u\}a i stopa mortal i teta od karci noma pl u\}a. I zbor parametara usl ovi I e su vi soka f rekvenci ja pojavq i vawa, i zra` ena di nami ka rasta i razvoja, kao i kratko prose no pre' i vqavaqe obol el i h od kar ci noma pl u\}a koje i znosi \{ est meseci do dve godi ne od trenutka postavqawa di jagnoze.

P rema popi su stanovni \{ tva i z 1991. godi ne Republ i - kog zavoda za stati sti ku, popul aci ju Rasi nskog okr uga -i ni 296.690 stanovni ka i to: 146.232 mu\{karaca i 150.458 ena. Prema i sti m podaci ma popul aci ju Central ne Srbi je -i ni 5.808.906 stanovni ka, pri -emu je u ovoj popul aci ji regi strovano 2.860.995 mu\{ karaca i 2.947.911 'ena.

I zvori podataka kori \{ \}eni u deskripti vnoj epi demi ol o\{koj studi ji u okvi ru ovog i stra' i vawa jesu regi star vi tal ni h dogal aja Republ i - kog zavoda za stati sti ku i P opul aci oni regi star za rak I nsti tuta za za\{ ti tu zdravq a Srbi je. Uvi dom u regi star vi tal ni h dogal aja Republ i - kog zavoda za stati sti ku dobi jeni su podaci o mortal i tetu od karci noma pl u\}a na podru-ju Rasi nskog okruga i Central ne Srbi je u peri odu od 1995. do 1999. godi ne, -i ju je f i nal nu obradu u f ormi stopa mortal i teta real i zoval a SI u’ ba za epi demi ol ogi ju i bi ostati st i -ka i stra` i vawa I nsti tuta za onkol ogi ju i radi ol ogi ju Srbi je 
Uvi dom u P opul aci oni regi star za rak I nsti tuta za za\{ ti tu zdravqa Srbi je, koji se vodi od 1996. godi ne, dobi jeni su podaci o morbi di tetu od karci noma pl u\}a na podru-ju Rasi nskog okruga i Central ne Srbi je u peri odu od 1996. do 2000. godi ne u obl i ku st opa i nci dence.

Deskri pti vni epi demi ol o\{ ki metod ukqu - vao je odre| i vawe pokazateq a obol evawa i umi rawa od kar ci noma pl u\}a na podr u-ju Rasi nskog okruga i Central ne Sr bi je u peri odu od 1995. do 2000. godi ne, kao i wi hove promene u f unkci ji vremena. Kao pokazateq i obol evawa kor i \{ \}ene su stope i nci dence (si rove i svetske) od kar ci noma pl u\}a za pomenuta podru-ja. Smrtnost od karci noma pl u\}a na pomenuti m teri tori jama predstavq ena je stopama mortal i teta (si rova i svetska), kao i meromu-estal osti umi rawa u pomenut i m popul aci jama $u$ i spi ti vanom per i odu. Sve nestandar di zovane (si rove) st ope st andar di zovane su po pol u, metodom di rektne standar di zaci je prema popul aci ji sveta, \{ to je omogu\}i l o otkl awawe uti caja razl i - te strukture i spi ti vane popul aci je prema pol u na vel i - nu stope i omogu\}i l o upore| i vawe doga| aja sa dr ugi m popul aci jama.

P ri menom sof tver skog paketa SPSS 8.0, i zra-unati su osnovni parametri deskri pti vne stati sti ke (di stri buci ja f rekvenci ja, mer e central ne tendenci je i var i jabi I i teta), zati mje testi rana zna ajnost $r$ azl i ke $u$ di stri buci ji u-estal osti stopa pri menom T-testa i anal i zom vari janse. Na osnovu vr ednost i stopa za svaku godi nu, do \{ I o se do jedna-i na trenda koji si mul i raju wi hovo kretawe za posmatrani peri od. Zna-ajnost trenda je procewena F-testom uz pomo kompjuter skog programa SPSS 8.0.

\section{Rezul t at i}

Si rova stopa i ndci dence karci nom pl u\}a za 'ene Rasi nskog okr uga pove\}al a se za 86,95 odsto u 2000. godi ni u onosu na 1996. godi nu (sa 4,6 na 8,6 na 100.000 stanovni ka), dok pove $a w e$ svet ske stope i nci dence za ovaj kar ci nom i znosi 56,09 odsto (sa 4,1 na 6,4 na 100.000 stanovni ka).

Model I i nearnog trenda za si rovu stopu i nci dence karci noma pl u\}a za 'ene Rasi nskog okr uga gl asi :

$$
\mathrm{Y}=3,32+1 \mathrm{~h} \text { godi ne, }
$$

a za svet sku stopu gl asi :

$$
\mathrm{Y}=2,54+0,76 \mathrm{~h} \text { godi ne. }
$$

Model I i nearnog trenda za 'ene Rasi nskog okr uga za si rovu i svetsku stopu i nci dence kar ci noma pl u\}a ukazuje na postojawe tendenci je stal nog por asta vrednost $i$ ovi $h$ stopa, \{ to pokazuju graf i koni 1 i 2.

Graf i kon 1. Li nearni trend si rove stope i nci dence kar ci noma pl u\}a kod 'ena Rasi nskog okr uga u peri odu od 1996. do 2000. godi ne.

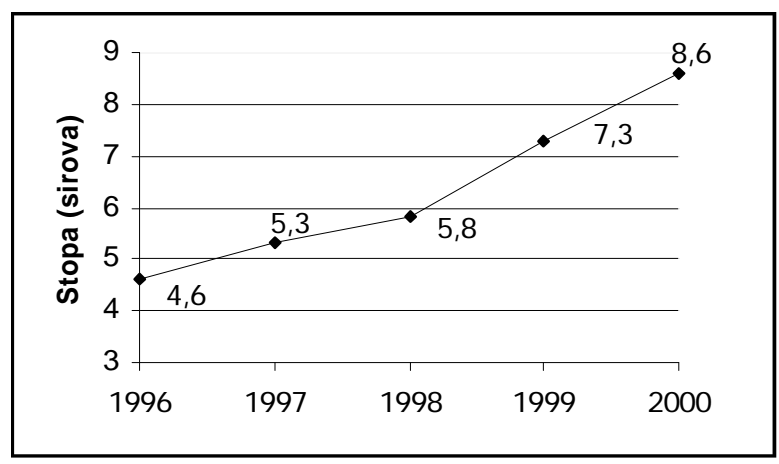

Graf i kon 2. Li nearni trend svetske stope i ncidence karci noma pl u\}a kod 'ena Rasi nskog okr uga u peri odu od 1996. do 2000. godi ne.

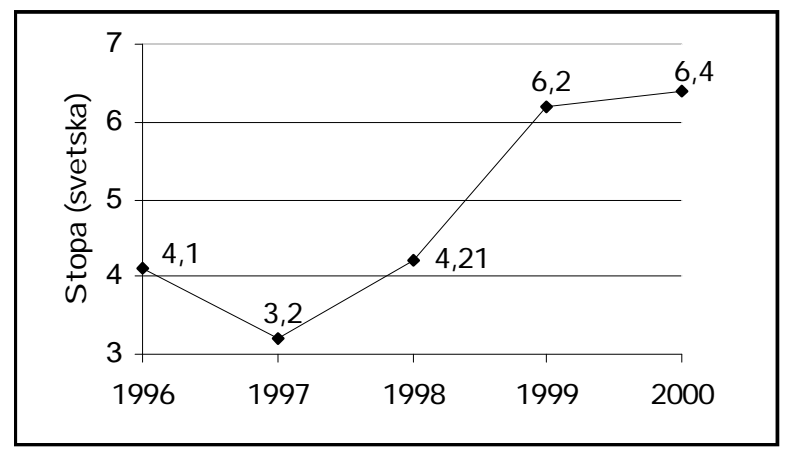

Si rova stopa mortal i teta od kar ci noma pl u\}a za `ensku popul aci ju Rasi nskog okruga pove\}al a se za 98,9 osto u 1999. godi ni u odnosu na 1995. godi nu (sa 8,22 na 15,35 na 100.000 st anovni ka), dok pove\}awe svetske sto- 
pe mortal i teta za ovaj kar ci nomi znosi 90,61 odsto (sa 4,58 na 8,73 na 100.000 stanovni ka).

Model I i nearnog trenda za si rovu stopu mortal i teta od karci noma pl u\}a za 'ene Rasi nskog okr uga gl asi :

$Y=4,329+2,41$ h godi ne,

a za svet sku stopu $\mathrm{gl}$ asi :

$\mathrm{Y}=3,09+1,15 \mathrm{~h}$ godi ne.

Ovaj model ukazuje na postojawe tendenci je stal nog porasta vrednosti si rove i svetske stope mortal i teta od karci noma pl u\}a me| u ' enskom popul aci jom Rasi nskog okr uga (graf i koni 3 i 4).

Graf i kon 3. Li nearni trend si rove stope mortal i teta od karci noma pl u\}a kod 'ena Rasi nskog okr uga u peri odu od 1995. do 1999. godi ne.

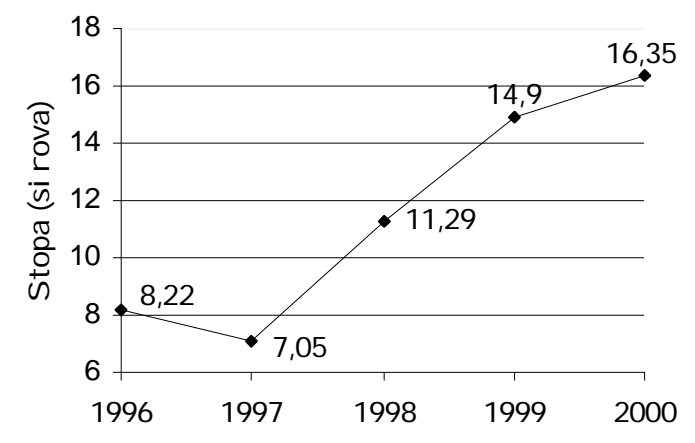

Graf i kon 4. Li nearni trend svetske mortal i teta od karci noma pl u\}a kod 'ena Rasi nskog okruga u peri odu od 1995. do 1999. godi ne.

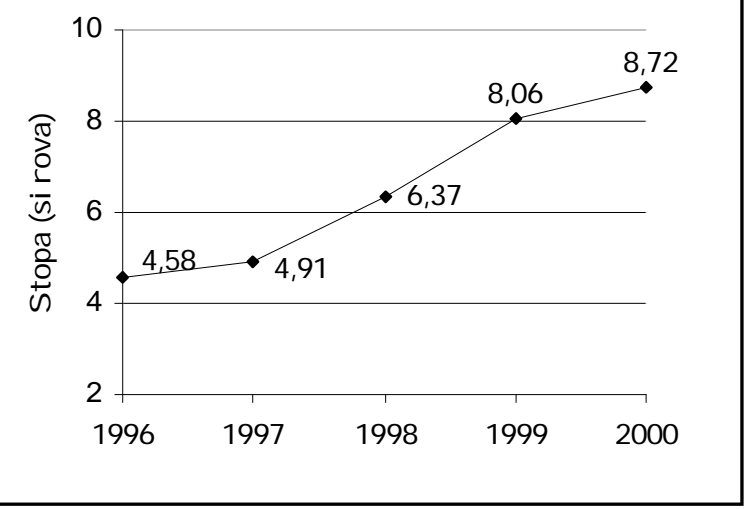

\section{Di skusi ja}

Si rova stopa i nci dence karci noma pl u\}a u enskoj popul aci ji Rasi nskog okruga pokazuje stat i sti - ki zna ajan trend por asta u peri odu od 1996. do 2000. godi ne (p je mawe od 0,01 ), kao i svet ska stopa, kod koje je p mawe od 0,05 (t abel a 1).

Tabel a 1. Parametri model a I i nearnog trenda si rove i svetske stope i nci dence kar ci noma pl u\}a za 'ene Rasi nskog okr uga u peri odu od 1996. do 2000. godi ne

\begin{tabular}{||l|c|c|c|c|c|c||}
\hline \hline $\begin{array}{l}\text { Stopa } \\
\text { i nci dence }\end{array}$ & $\mathbf{R}^{2}$ & $\mathbf{d f}$ & $\mathbf{F}$ & $\mathbf{p}$ & $\mathbf{b}_{0}$ & $\mathbf{b}_{1}$ \\
\hline Si rova & 0,959 & 3 & 70,9 & 0,004 & 3,3200 & 1,000 \\
\hline Svetska & 0,730 & 3 & 8,10 & 0,035 & 2,5420 & 0,760 \\
\hline \hline
\end{tabular}

Si rova stopa mortal i teta od karci noma pl u\}a kod 'ena Rasi nskog okruga pokazuje stati st i - ki zna ajan trend por asta u per i odu od 1995. do 1999. godi ne (p je mawe od $0,05)$, kao i svet ska stopa kod koje je p mawe od 0,01 (t abel a 2).

Tabel a 2. Parametri model a I i nearnog trenda si rove i svetske stope mortal i teta od karci noma pl u\}a za 'ene Rasi nskog okr uga u peri odu od 1995. do 1999. god.

\begin{tabular}{||l|c|c|c|c|c|c||}
\hline \hline $\begin{array}{l}\text { Stopa } \\
\text { i nci dence }\end{array}$ & $\mathbf{R}^{2}$ & $\mathbf{d f}$ & $\mathbf{F}$ & $\mathbf{p}$ & $\mathbf{b}_{0}$ & $\mathbf{b}_{1}$ \\
\hline Si rova & 0,885 & 3 & 23,13 & 0,017 & 4,329 & 2,4110 \\
\hline Svetska & 0,962 & 3 & 75,18 & 0,003 & 3,095 & 1,1450 \\
\hline \hline
\end{tabular}

\section{Zakqurak}

1. Si rova stopa i nci dence karci noma pl u\}a u enskoj popul aci ji Rasi nskog okruga pokazuje stati sti -ki zna-ajan trend por asta u peri odu od 1996. do 2000. godi ne ( $p$ je mawe od 0,01), kao i svetska stopa kod koje je p mawe od 0,05.

2. Si rova stopa mortal i teta od karci noma pl u\}a u enskoj popul aci ji Rasi nskog okruga pokazuje stati sti - ki zna-ajan trend por asta u peri odu od 1995. do 1999. godi ne ( $p$ je mawe od 0,05 ), kao i svet ska stopa kod koje je p mawe od 0,01. 


\section{Lit erat ura}

1. I or|evi \} M., Mitrovi \} N.: Mal i gna oboq ewa u Srbiji krajem XX veka, Beograd: Yugoslaviapublic, 1995

2. Fift fac tfrom the World Health Report 1998. (Global Health situation and trends 1995-2025.). Geneva: WH0, 1998 Available from URL: http://www.who.int. 